\title{
A Bipartite Strengthening of the Crossing Lemma
}

\author{
Jacob Fox ${ }^{1, \star}$, János Pach $^{2, \star \star}$, and Csaba D. Tóth ${ }^{3}$ \\ ${ }^{1}$ Department of Mathematics, Princeton University, Princeton, NJ, USA \\ jacobfox@math.princeton.edu \\ ${ }^{2}$ City College, CUNY and Courant Institute, NYU, New York, NY, USA \\ pach@cims.nyu.edu \\ ${ }^{3}$ University of Calgary, Calgary, AB, Canada \\ cdtoth@ucalgary.ca
}

\begin{abstract}
The celebrated Crossing Lemma states that, in every drawing of a graph with $n$ vertices and $m \geq 4 n$ edges there are at least $\Omega\left(\mathrm{m}^{3} / \mathrm{n}^{2}\right)$ pairs of crossing edges; or equivalently, there is an edge that crosses $\Omega\left(\mathrm{m}^{2} / \mathrm{n}^{2}\right)$ other edges. We strengthen the Crossing Lemma for drawings in which any two edges cross in at most $O(1)$ points.

We prove for every $k \in \mathbb{N}$ that every graph $G$ with $n$ vertices and $m \geq 3 n$ edges drawn in the plane such that any two edges intersect in at most $k$ points has two disjoint subsets of edges, $E_{1}$ and $E_{2}$, each of size at least $c_{k} m^{2} / n^{2}$, such that every edge in $E_{1}$ crosses all edges in $E_{2}$, where $c_{k}>0$ only depends on $k$. This bound is best possible up to the constant $c_{k}$ for every $k \in \mathbb{N}$. We also prove that every graph $G$ with $n$ vertices and $m \geq 3 n$ edges drawn in the plane with $x$-monotone edges has disjoint subsets of edges, $E_{1}$ and $E_{2}$, each of size $\Omega\left(m^{2} /\left(n^{2}\right.\right.$ polylog $\left.\left.n\right)\right)$, such that every edge in $E_{1}$ crosses all edges in $E_{2}$. On the other hand, we construct $x$-monotone drawings of bipartite dense graphs where the largest such subsets $E_{1}$ and $E_{2}$ have size $O\left(m^{2} /\left(n^{2} \log (m / n)\right)\right)$.
\end{abstract}

\section{Introduction}

The crossing number $\operatorname{cr}(G)$ of a graph 11 is the minimum number of crossings in a drawing of $G$. A drawing of a graph $G$ is a planar embedding which maps the vertices to distinct points in the plane and each edge to a simple continuous arc connecting the corresponding vertices but not passing through any other vertex. A crossing is a pair of curves and a common interior point between the two curves (the intersections at endpoints or vertices do not count as crossings). A celebrated result of Ajtai et al. ACNS82 and Leighton [L84, known as

\footnotetext{
* Research supported by an NSF Graduate Research Fellowship and a Princeton Centennial Fellowship.

** Supported by NSF Grant CCF-05-14079, and by grants from NSA, PSC-CUNY, Hungarian Research Foundation OTKA, and BSF.

${ }^{1}$ The graphs considered here are simple, having no loops or parallel edges.
} 
the Crossing Lemma, states that the crossing number of every graph $G$ with $n$ vertices and $m \geq 4 n$ edges satisfies

$$
\operatorname{cr}(G)=\Omega\left(\frac{m^{3}}{n^{2}}\right) .
$$

The best known constant coefficient is due to PRTT06. Leighton L84 was motivated by applications to VLSI design. Szekély [S97] used the Crossing Lemma to give simple proofs of Szemerédi-Trotter bound on the number of point-line incidences ST83, a bound on Erdős's unit distance problem and Erdős's distinct distance problem [E46]. The Crossing Lemma has since found many important applications, in combinatorial geometry D98, KT04, PS98, PT02, STT02, and number theory [ENR00, TV06].

The pairwise crossing number pair-cr $(G)$ of a graph $G$ is the minimum number of pairs of crossing edges in a drawing of $G$. The lower bound (1) also holds for the pairwise crossing number with the same proof. It follows that in every drawing of a graph with $n$ vertices and $m \geq 4 n$ edges, there is an edge that crosses at least $\Omega\left(\mathrm{m}^{2} / \mathrm{n}^{2}\right)$ other edges. Conversely, if in every drawing of every graph with $m \geq 3 n$ edges some edge crosses $\Omega\left(m^{2} / n^{2}\right)$ others, then we have pair-cr $(G)=\Omega\left(m^{3} / n^{2}\right)$ for every graph $G$ with $m \geq 4 n$ edges. Indeed, by successively removing edges that cross many other edges, we obtain the desired lower bound for the total number of crossing pairs. In this note, we prove a bipartite strengthening of this result for drawings where any two edges intersect in at most a constant number of points.

Theorem 1. For every $k \in \mathbb{N}$, there is a constant $c_{k}>0$ such that for every drawing of a graph $G=(V, E)$ with $n$ vertices and $m \geq 3 n$ edges, no two of which intersect in more than $k$ points, there are disjoint subsets $E_{1}, E_{2} \subset E$, each of size at least $c_{k} m^{2} / n^{2}$, such that every edge in $E_{1}$ crosses all edges in $E_{2}$.

We have $k=1$ in straight-line drawings, $k=(\ell+1)^{2}$ if every edge is a polyline with up to $\ell$ bends, and $k=d^{2}$ if the edges are sufficiently generic algebraic curves (e.g., splines) of degree at most $d$. Note also that every graph $G$ has a drawing with $\operatorname{cr}(G)$ crossings in which any two edges cross at most once [V05].

The dependence on $k$ in Theorem 1 is necessary: We show that one cannot expect bipartite crossing families of edges of size $\Omega\left(\mathrm{m}^{2} / \mathrm{n}^{2}\right)$ if any two edges may cross arbitrarily many times, even if the graph drawings are restricted to be $x$-monotone. An $x$-monotone curve is a continuous arc that intersects every vertical line in at most one point. A drawing of a graph is $x$-monotone if every edge is mapped to an $x$-monotone curve.

Theorem 2. For every $n, m \in \mathbb{N}$ with $m \leq n^{2} / 4$, there is a bipartite graph $G=(V, E)$ with $n$ vertices, $m$ edges, and an $x$-monotone drawing such that any two disjoint subsets $E_{1}, E_{2} \subset E$ of equal size $\left|E_{1}\right|=\left|E_{2}\right|=t$, where every edge in $E_{1}$ crosses all edges in $E_{2}$, satisfy

$$
t=O\left(\frac{m^{2}}{n^{2} \log (m / n)}\right) .
$$


We present the tools used for the bipartite strengthening of the Crossing Lemma in the next section. Theorem 1 is proved in Section 3. Our construction of $x$ monotone drawings are discussed in Section 4 . Finally, Section 5 contains a weaker analogue of Theorem 1 for $x$-monotone drawings and a further strengthening of the Crossing Lemma for graphs satisfying some monotone property.

\section{Tools}

The proof of Theorem 1 relies on a recent result on the intersection pattern of $k$-intersecting curves. For a collection $C$ of curves in the plane, the intersection graph is defined on the vertex set $C$, two elements of $C$ are adjacent if the (relative) interiors of the corresponding curves intersect. A complete bipartite graph is balanced if the vertex classes differ in size by at most one. For brevity, we call a balanced complete bipartite graph a bi-clique.

Theorem 3. FPT07a] Given $m$ curves in the plane such that at least [ $^{2}$ pairs intersect and any two curves intersect in at most $k$ points, their intersection graph contains a bi-clique with at least $c_{k} \varepsilon^{64} m$ vertices where $c_{k}>0$ depends only on $k$.

If follows from the Crossing Lemma that in every drawing of a dense graph, the intersection graph of the edges is also dense. Therefore, Theorem 3 implies Theorem 1 in the special case that $G$ is dense. This connection was first observed by Pach and Solymosi PS01 who proved Theorem 1 for straight-line drawings of dense graphs.

If a graph $G$ is not dense, we decompose $G$ recursively into induced subgraphs with an algorithm reminiscent of PST00 until one of the components is dense enough so that Theorem [3, like before, implies Theorem 11. The decomposition algorithm successively removes bisectors, and we use Theorem 4 below to keep the total number of deleted edges under control.

The bisection width, denoted by $b(G)$, is defined for every simple graph $G$ with at least two vertices. It is the smallest nonnegative integer such that there is a partition of the vertex set $V=V_{1} \cup^{*} V_{2}$ with $\frac{1}{3} \cdot|V| \leq V_{i} \leq \frac{2}{3} \cdot|V|$ for $i=1,2$, and $\left|E\left(V_{1}, V_{2}\right)\right|=b(G)$. Pach, Shahrokhi, and Szegedy [PSS96] gave an upper bound on the bisection width in terms of the crossing number and the $L_{2}$-norm of the degree vector (it is an easy consequence of the weighted version of the famous Lipton-Tarjan separator theorem [LT79, GM90]).

Theorem 4. PSS96] Let $G$ be a graph with $n$ vertices of degree $d_{1}, d_{2}, \ldots, d_{n}$. Then

$$
b(G) \leq 10 \sqrt{\operatorname{cr}(G)}+2 \sqrt{\sum_{i=1}^{n} d_{i}^{2}(G)} .
$$

\section{Proof of Theorem 1}

Let $G=(V, E)$ be a graph with $n$ vertices and $m \geq 3 n$ edges. Since a graph with more than $3 n-6$ edges cannot be planar, it must have crossing edges. Hence, as 
long as $3 n \leq m<10^{6} n$, Theorem 11 holds with $\left|E_{1}\right|=\left|E_{2}\right|=1 \geq 10^{-12} m^{2} / n^{2}$. We assume $m \geq 10^{6} n$ in the remainder of the proof.

Let $D$ be a drawing of $G$. To use the full strength of Theorem 4 , we transform the drawing $D$ into a drawing $D^{\prime}$ of a graph $G^{\prime}=\left(V^{\prime}, E^{\prime}\right)$ with $m$ edges, at most $2 n$ vertices, and maximum degree at most $\lceil 2 m / n\rceil$, so that the intersection graph of $E^{\prime}$ is isomorphic to that of $E$. If the degree of a vertex $v \in V$ is above the average degree $\bar{d}=2 \mathrm{~m} / n$, split $v$ into $\lceil d / \bar{d}\rceil$ vertices $v_{1}, \ldots, v_{\lceil d / \bar{d}\rceil}$ arranged along a circle of small radius centered at $v$. Denote the edges of $G$ incident to $v$ by $\left(v, w_{1}\right), \ldots,\left(v, w_{d}\right)$ in clockwise order in the drawing $D$. In $G^{\prime}$, connect $w_{j}$ with $v_{i}$ if and only if $\bar{d}(i-1)<j \leq \bar{d} i$, where $1 \leq j \leq d$ and $1 \leq i \leq\lceil d / \bar{d}\rceil$. Two edges of $G^{\prime}$ cross if and only if the corresponding edges of $G$ cross. Also, letting $d(v)$ denote the degree of vertex $v$ in $G^{\prime}$, the number of vertices of $G^{\prime}$ is

$$
\sum_{v \in V}\lceil d(v) / \bar{d}\rceil<\sum_{v \in V} 1+d(v) / \bar{d}=2 n .
$$

Hence the resulting $G^{\prime}$ and $D^{\prime}$ have all the required properties.

We will decompose $G^{\prime}$ recursively into induced subgraphs until each induced subgraph is either a singleton or it has so many pairs of crossing edges that Theorem 3 already implies Theorem 1. Theorem 3 implies that the intersection graph of the edges of an induced subgraph $H$ of $G^{\prime}$ contains a bi-clique of size at least $c_{k}\left(\frac{p(H)}{e(H)^{2}}\right)^{64} e(H)$, where $p(H)$ is the number of pairs of crossing edges in $H$ in the drawing $D^{\prime}, e(H)$ is the number of edges of $H$, and $c_{k}>0$ is the constant depending on $k$ only in Theorem 3. So the intersection graph of the edge set of $G^{\prime}$ (and hence also of $G$ ) contains a bi-clique of size $\Omega_{k}\left(\mathrm{~m}^{2} / \mathrm{n}^{2}\right.$ ) if there is an induced subgraph $H$ of $G^{\prime}$ with

$$
\varepsilon_{k} \frac{m^{2}}{n^{2}} \leq\left(\frac{p(H)}{e(H)^{2}}\right)^{64} e(H),
$$

where $\varepsilon_{k}>0$ is any constant depending on $k$ only. We use $\varepsilon_{k}=\left(10^{9} k\right)^{-64}$ for convenience. Assume, to the contrary, that (3) does not hold for any induced subgraph $H$ of $G^{\prime}$.

Every induced subgraph $H$ has at most $k p(H)$ crossings in the drawing $D^{\prime}$, hence $\operatorname{cr}(H) \leq k p(H)$. It is enough to find an induced subgraph $H$ for which

$$
\frac{e(H)^{2-1 / 64}}{10^{9}}\left(\frac{m}{n}\right)^{\frac{1}{32}} \leq \operatorname{cr}(H),
$$

since this combined with $\operatorname{cr}(H) \leq k p(H)$ implies (3).

Next, we decompose the graph $G^{\prime}$ of at most $2 n$ vertices and $m$ edges with the following algorithm.

\section{Decomposition Algorithm}

1. Let $S_{0}=\left\{G^{\prime}\right\}$ and $i=0$.

2. While $(3 / 2)^{i} \leq 4 n^{2} / m$ and no $H \in S_{i}$ that satisfies (4), do

Set $i:=i+1$. Let $S_{i}:=\emptyset$. For every $H \in S_{i-1}$, do 
- If $|V(H)| \leq(2 / 3)^{i} 2 n$, then let $S_{i}:=S_{i} \cup\{H\}$;

- otherwise split $H$ into induced subgraphs $H_{1}$ and $H_{2}$ along a bisector 3. Return $S_{i}$. of size $b(H)$, and let $S_{i}:=S_{i} \cup\left\{H_{1}, H_{2}\right\}$.

For every $i$, every graph $H \in S_{i}$ satisfying the end condition has at most $|V(H)| \leq(2 / 3)^{i} 2 n$ vertices. Hence, the algorithm terminates in $t \leq \log _{(3 / 2)} 2 n$ rounds and it returns a set $S_{t}$ of induced subgraphs. Let $T_{i} \subset S_{i}$ be the set of those graphs in $S_{i}$ that have more than $(2 / 3)^{i} 2 n$ vertices. Notice that $\left|T_{i}\right| \leq$ $(3 / 2)^{i}$. Denote by $G_{i}$ the disjoint union of the induced subgraphs in $S_{i}$.

We use Theorem 4 for estimating the number of edges deleted throughout the decomposition algorithm. Substituting the upper bound for $\operatorname{cr}(H)$ and using Jensen's inequality for the concave function $f(x)=x^{1-1 / 128}$, we have for every $i=0,1, \ldots, t$,

$$
\begin{aligned}
\sum_{H \in T_{i}} \sqrt{\operatorname{cr}(H)} & \leq \sum_{H \in T_{i}} \sqrt{\frac{e(H)^{2-1 / 64}}{10^{9}}\left(\frac{m}{n}\right)^{\frac{1}{32}}}=10^{-\frac{9}{2}}\left(\frac{m}{n}\right)^{\frac{1}{64}} \sum_{H \in T_{i}} e(H)^{1-\frac{1}{128}} \\
& \leq 10^{-\frac{9}{2}}\left(\frac{m}{n}\right)^{\frac{1}{64}}\left|T_{i}\right|^{\frac{1}{128}} m^{1-\frac{1}{128}} \leq 10^{-\frac{9}{2}}\left(\frac{3}{2}\right)^{\frac{i}{128}} \frac{m^{1+1 / 128}}{n^{1 / 64}} .
\end{aligned}
$$

Denoting by $d(v, H)$ the degree of vertex $v$ in an induced subgraph $H$, we have

$$
\begin{aligned}
\sum_{H \in T_{i}} \sqrt{\sum_{v \in V(H)} d^{2}(v, H)} & \leq \sqrt{\left|T_{i}\right|} \sqrt{\sum_{v \in V\left(G_{i}\right)} d^{2}\left(v, G_{i}\right)} \\
& \leq \sqrt{(3 / 2)^{i}} \sqrt{n \cdot(\bar{d})^{2}} \leq \frac{2 m}{\sqrt{n}} \sqrt{(3 / 2)^{i}}
\end{aligned}
$$

In the first of the two above inequalities, we use the Cauchy-Schwartz inequality to get $\sum_{H \in T_{i}} \sqrt{x_{H}} \leq \sqrt{\left|T_{i}\right|} \sqrt{\sum_{H \in T_{i}} x_{H}}$ with $x_{H}=\sum_{v \in V(H)} d^{2}(v, H)$.

By Theorem 4 the total number of edges deleted during this process is

$$
\begin{aligned}
\sum_{i=0}^{t-1} \sum_{H \in T_{i}} b(H) & \leq 10 \sum_{i=0}^{t-1} \sum_{H \in T_{i}} \sqrt{\operatorname{cr}(H)}+2 \sum_{i=0}^{t-1} \sum_{H \in T_{i}} \sqrt{\sum_{v \in V(H)} d^{2}(v, H)} \\
& \leq 10^{-\frac{7}{2}} \frac{m^{1+1 / 128}}{n^{1 / 64}} \sum_{i=0}^{t-1}(3 / 2)^{\frac{i}{128}}+4 \frac{m}{\sqrt{n}} \sum_{i=0}^{t-1} \sqrt{(3 / 2)^{i}} \\
& \leq \frac{m^{1+1 / 128}}{4 n^{1 / 64}}\left(\frac{n^{2}}{m}\right)^{1 / 128}+100 m^{1 / 2} n^{1 / 2} \leq \frac{m}{2} .
\end{aligned}
$$

The second inequality uses the earlier upper bounds for $\sum_{H \in T_{i}} \sqrt{\operatorname{cr}(H)}$ and $\sum_{H \in T_{i}} \sqrt{\sum_{v \in V(H)} d^{2}(v, H)}$, the third inequality uses the geometric series formula and the upper bound $t \leq \log _{(3 / 2)} 2 n$, while the last inequality follows from the fact that $m \geq 10^{6} n$. 
So at least $m / 2$ edges survive and each of the induced subgraphs in $S_{t}$ has at most $(2 / 3)^{t} 2 n \leq 2 n /\left(4 n^{2} / m\right)=m / 2 n$ vertices. Also $G^{\prime}$ has at most $2 n$ vertices, so using Jensen's inequality for the convex function $g(x)=\left(\begin{array}{l}x \\ 2\end{array}\right)$, the total number of vertex pairs lying in a same induced subgraph of $S_{t}$ is less than

$$
\frac{2 n}{m / 2 n} \frac{(m / 2 n)^{2}}{2}=\frac{m}{2} \text {, }
$$

a contradiction. We conclude that the decomposition algorithm must have found an induced subgraph $H$ satisfying (44). This completes the proof of Theorem[1

\section{Drawings with Edges as $x$-monotone Curves}

It is known that Theorem 3 does not hold without the assumption that any two curves intersect in at most a constant number of points. Using a construction from [F06], Pach and G. Tóth [PT06] constructed for every $n \in \mathbb{N}$, a collection of $n x$-monotone curves whose intersection graph is dense but every bi-clique it contains has at most $O(n / \log n)$ vertices. Theorem 2 shows a stronger construction holds: the curves are edges in an $x$-monotone drawing of a dense bipartite graph, where $\Theta\left(n^{2}\right)$ curves have only $n$ distinct endpoints.

The proof of Theorem 3 builds on a crucial observation: Golumbic et al. GRU83 noticed a close connection between intersection graphs of $x$-monotone curves and partially ordered sets. Consider $n$ continuous functions $f_{i}:[0,1] \rightarrow \mathbb{R}$. The graph of every continuous real function is clearly an $x$-monotone curve. Define the partial order $\prec$ on the set of functions by $f_{i} \prec f_{j}$ if and only if $f_{i}(x)<f_{j}(x)$ for all $x \in[0,1]$. Two $x$-monotone curves intersect if and only if they are incomparable under this partial order $\prec$.

Lemma 1. GRU83. The elements of any partially ordered $\operatorname{set}(\{1,2, \ldots, n\}, \prec)$ can be represented by continuous real functions $f_{1}, f_{2}, \ldots, f_{n}$ defined on the interval $[0,1]$ such that $f_{i}(x)<f_{j}(x)$ for every $x$ if and only if $i \prec j(i \neq j)$.

Proof. Let $(\{1,2, \ldots, n\}, \prec)$ be a partial order, and let $\Pi$ denote the set consisting of all of its extensions $\pi(1) \prec \pi(2) \prec \ldots \prec \pi(n)$ to a total order. Clearly, every element of $\Pi$ is a permutation of the numbers $1,2, \ldots, n$. Let $\pi_{1}, \pi_{2}, \ldots, \pi_{t}$ be an arbitrary labeling of the elements of $\Pi$. Assign distinct points $x_{k} \in[0,1]$ to each $\pi_{k}$ such that $0=x_{1}<x_{2}<\ldots<x_{t}=1$. For each $i(1 \leq i \leq n)$, define a continuous, piecewise linear function $f_{i}(x)$, as follows. For any $k(1 \leq k \leq t)$, set $f_{i}\left(x_{k}\right)=\pi_{k}^{-1}(i)$, and let $f_{i}(x)$ be linear over each interval $\left[x_{k}, x_{k+1}\right]$.

Obviously, whenever $i \prec j$ for some $i \neq j$, we have that $\pi_{k}^{-1}(i) \prec \pi_{k}^{-1}(j)$ for every $k$, and hence $f_{i}(x)<f_{j}(x)$ for all $x \in[0,1]$. On the other hand, if $i$ and $j$ are incomparable under the partial order $\prec$, there are indices $k$ and $k^{\prime}$ $\left(1 \leq k \neq k^{\prime} \leq m\right)$ such that $f_{i}\left(x_{k}\right)<f_{j}\left(x_{k}\right)$ and $f_{i}\left(x_{k}\right)>f_{j}\left(x_{k^{\prime}}\right)$, therefore, by continuity, the graphs of $f_{i}$ and $f_{j}$ must cross at least once in the interval $\left(x_{k}, x_{k^{\prime}}\right)$. This completes the proof. 
The following lemma is the key for the proof of Theorem 2 It presents a partially ordered set of size $n^{2}$ whose incomparability graph contains bi-cliques of size at most $O\left(n^{2} / \log n\right)$, yet it can be represented with $x$-monotone curves having only $2 n$ endpoints.

Lemma 2. For every $n \in \mathbb{N}$, there is a partially ordered set $P$ with $n^{2}$ elements satisfying the following properties

1. every bi-clique in the incomparability graph of $P$ has size at most $O\left(n^{2} / \log n\right)$,

2. there are equitable partitions $P=P_{1} \cup \ldots \cup P_{n}$ and $P=Q_{1} \cup \ldots \cup Q_{n}$ such that

(a) for each $i$, there is a linear extension of $P$ where the elements of $P_{i}$ are consecutive,

(b) there is a linear extension of $P$ where the elements of each $Q_{j}$ are consecutive, and

(c) for every $i$ and $j$, we have $\left|P_{i} \cap Q_{j}\right|=1$.

We now prove Theorem 2, pending the proof of Lemma 2, Note that it suffices to prove Theorem 2 in the case $m=n^{2} / 4$, that is, when $G$ is a bi-clique. By deleting some of the edges of this construction, we obtain a construction for every $m \leq n^{2} / 4$, since edge deletions also decrease the intersection graph of the edges. So it is enough to prove the following.

Lemma 3. There is an $x$-monotone drawing of $K_{n, n}$ such that every bi-clique in the intersection graph of the edges has size at most $O\left(n^{2} / \log n\right)$.

Proof. Let $P$ be a poset described in Lemma 2. Represent $P$ with $x$-monotone curves as in the proof of Lemma 1 such that the last linear extension $\pi_{t}$ has property (b) of Lemma 2, that is, the elements of each $Q_{j}$ are consecutive in $\pi_{t}$.

We transform the $n^{2} x$-monotone curves representing $P$ into an $x$-monotone drawing of $K_{n, n}$. We introduce two vertex classes, each of size $n$, as follows. Along the line $x=1$, the right endpoints of the $x$-monotone curves in each $Q_{j}$ are consecutive. Introduce a vertex on $x=1$ for each $Q_{j}$, and make it the common right endpoint of all curves in $Q_{j}$ by deforming the curves over the interval $\left(x_{t-1}, 1\right]$ but keeping their intersection graph intact. These $n$ vertices along the line $x=1$ form one vertex class of $K_{n, n}$.

For each $i$, there is a vertical line $x=x_{i}$ along which the $x$-monotone curves in $P_{i}$ are consecutive. Introduce a vertex for each $P_{i}$ on line $x=x_{i}$, and make it the common left endpoint of all curves in $P_{i}$ by deforming the curves over the interval $\left[x_{i}, x_{i+1}\right)$ and erasing their portion over the interval $\left[0, x_{i}\right)$. These $n$ vertices, each lying on a line $x=x_{i}$, form the second vertex class of $K_{n, n}$. After truncating and slightly deforming the $n^{2}$ curves representing $P$, we have constructed an $x$-monotone drawing of $K_{n, n}$.

Note that the intersection graph of the edges of this drawing of $K_{n, n}$ is a subgraph of the incomparability graph of $P$, so every bi-clique of the intersection graph of the edges has size at most $O\left(n^{2} / \log n\right)$. 
Proof of Lemma 2. We start out with introducing some notation for directed graphs. For a subset $S$ of vertices in a directed graph $G$, let $N_{+}(S)$ denote the set of vertices $x$ in $G$ such that there is a vertex $s \in S$ with an edge $(s, x)$ in $G$. Similarly, $N_{-}(S)$ is the set of vertices $y$ in $G$ such that there is a vertex $s \in S$ with an edge $(y, s)$ in $G$. A directed graph has path-girth $k$ if $k$ is the smallest positive integer for which there are vertices $x$ and $y$ having at least two distinct walks of length $k$ from $x$ to $y$. Equivalently, denoting the adjacency matrix of $G$ by $A_{G}$, it has path-girth $k$ if $A_{G}^{1}, \ldots, A_{G}^{k-1}$ are all $0-1$ matrices, but the matrix $A_{G}^{k}$ has an entry greater than 1 .

A directed graph $H=(X, E)$ is an $\epsilon$-expander if both $N_{+}(S)$ and $N_{-}(S)$ has size at least $(1+\epsilon)|S|$ for all $S \subset V$ with $1 \leq|S| \leq|V| / 2$. An expander is a directed graph with constant expansion.

We will use that for every $v \in \mathbb{N}$, there is a constant degree expander with $v$ vertices and path-girth $\Omega(\log v)$. This can be proved by a slight alteration of a random constant degree directed graph. We suppose for the remainder of the proof that $H=(X, E)$ is an $\epsilon$-expander with $v$ vertices, maximal degree at most $d$, and path-girth greater than $c \log v$, where $\epsilon, c$, and $d$ are fixed positive constants.

For every $a \in \mathbb{N}$, we define a poset $P(a, H)$ with ground set $X \times\{1,2, \ldots, a\}$, generated by the relations $\left(j_{1}, k_{1}\right) \prec\left(j_{2}, k_{2}\right)$ whenever $k_{2}=k_{1}+1$ and $\left(j_{1}, j_{2}\right)$ is an edge of $H$.

Let $P_{0}=P(a, H)$ with $a=\left\lfloor\min \left(c,(10 \log d)^{-1}\right) \cdot \log v\right\rfloor$. One can show, by essentially the same argument as in [F06], that the partially ordered set $P_{0}$ has the following three properties.

1. $P_{0}$ has $a|X|=\Theta(v \log v)$ elements,

2. each element of $P_{0}$ is comparable with fewer than $d^{a} \leq v^{1 / 10}$ other elements of $P_{0}$, and

3. the largest bi-clique in the incomparability graph of $P_{0}$ has size at most $O(|X|)=O(v)$.

Since the path-girth of $H$ is greater than $a$, if $x, y, z, w \in P_{0}$ satisfy both $w \prec y \prec x$ and $w \prec z \prec x$, then $y$ and $z$ must be comparable. That is, the poset in Figure 1(a) cannot be a subposet of $P_{0}$. The poset $P$ required for Lemma 2 will be a linear size subposet of $P_{0}$. We next describe the construction of $P$.

A chain is a set of pairwise comparable elements. The maximum chains in $P_{0}$ each have size $a$, having one element from each of $X \times\{i\}, i=1,2, \ldots, a$. Greedily choose as many disjoint chains of size $a$ as possible from $P_{0}$, denote the set of chains by $\mathcal{C}=\left\{C_{1}, \ldots, C_{w}\right\}$, where $w$ is the number of chains. By the expansion property of $H$, we have $w=\Theta(|X|)=\Theta(v)$.

We choose greedily disjoint subsets $P_{1}, \ldots, P_{h a}$ of $P$, each of which is the union of $h=\Theta(\sqrt{v})$ chains of $\mathcal{C}$. Each $P_{i}$ has the property that, besides the comparable pairs within each of the the $h$ chains, there are no other comparable pairs in $P_{i}$. We can choose the $h$ chains of each $P_{i}$ greedily: after choosing the $k^{\text {th }}$ chain in $P_{i}$, we have to choose the $(k+1)^{\text {th }}$ chain such that none of its 


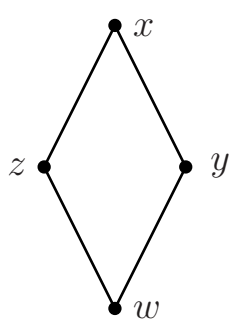

(a)

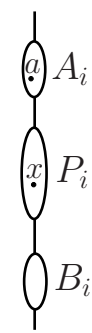

(b)

Fig. 1. (a) The Hasse diagram of a four element excluded subposet of $P_{0}$. (b) A linear extension of $P$ where $B_{i} \prec P_{i} \prec A_{i}$.

elements are comparable with any element of the first $k$ chains of $P_{i}$. Since at most $k a v^{1 / 10} \leq h a v^{1 / 10}=v^{3 / 5+o(1)}$ of the $w-(i-1) h-k=\Theta(v)$ remaining chains contain an element comparable with the first $k$ chains of $P_{i}$, almost any of the remaining chains can be chosen as the $(k+1)^{\text {th }}$ chain of $P_{i}$. Finally, let $P=P_{1} \cup \ldots \cup P_{h a}$. As mentioned earlier, we have $|P|=\Theta\left(\left|P_{0}\right|\right)$, and the largest bi-clique in the incomparability graph of $P$ is of size $O\left(\left|P_{0}\right| / \log \left|P_{0}\right|\right)=$ $O(|P| / \log |P|)$.

Since the poset in Fig. 1(a) is not a subposet of $P_{0}$, no element of $P_{0} \backslash C_{k}$, $C_{k} \in \mathcal{C}$, can be both greater than an element of $C_{k}$ and less than another element of $C_{k}$. By construction, if two elements of $P_{i}$ are comparable, then they belong to the same chain. Therefore, no element of $P \backslash P_{i}$ can be both greater than an element of $P_{i}$ and less than another element of $P_{i}$.

Consider the partition $P=A_{i} \cup P_{i} \cup B_{i}$, where an element $a \in P \backslash P_{i}$ is in $A_{i}$ if and only if there is an element $x \in P_{i}$ such that $x \prec a$. There is a linear extension of $P$ in which the elements of $A_{i}$ are the largest, followed by the elements of $P_{i}$, and the elements of $B_{i}$ are the smallest (see Fig. 11(b)). This is because no element of $P \backslash P_{i}$ can be both greater than an element of $P_{i}$ and less than another element of $P_{i}$.

Partition $P$ into subsets $P=X_{1} \cup \ldots \cup X_{a}$, where $X_{j}$ consists of the elements $(j, x) \in P$ with $x \in X$. Each $X_{j}$ contains exactly $h^{2} a$ elements, $h$ elements from each $P_{i}$. Arbitrarily partition each $X_{j}$ into $h$ sets $X_{j}=Q_{(j-1) h+1} \cup \ldots \cup Q_{j h}$ such that each $Q_{k}$ contains one element from each $P_{i}$. Since the elements in each $X_{j}$ form an antichain (a set of pairwise incomparable elements), any linear order of the elements of $P$ for which the elements of $X_{j}$ are smaller than the elements of $X_{k}$ for $1 \leq j<k \leq a$ is a linear extension of $P$. Hence, there is a linear extension of $P$ such that, for each $j$, the elements of every $Q_{j}$ are consecutive.

We have established that $P$ has all the desired properties. We can choose $v$ such that $n \leq h a$ and $h a=O(n)$, so $v=\Theta\left(n^{2} / \log n\right)$. If $h a$ is not exactly $n$, we may simply take the subposet whose elements are $\left(P_{1} \cup \ldots \cup P_{n}\right) \cap\left(Q_{1} \cup \ldots \cup Q_{n}\right)$. This completes the proof of Lemma 2 . 


\section{Concluding Remarks}

We can prove a weaker form of Theorem 1 for $x$-monotone curves, since our main tools (Theorems 3 and 4) are available in weaker forms in this case. It was recently shown in FPT07b that there is a constant $c>0$ such that the intersection graph $G$ of any $n x$-monotone curves, at least $\varepsilon n^{2}$ pairs of which intersect, contains a bi-clique with at least $c \varepsilon^{2} n /\left(\log \frac{1}{\varepsilon} \log n\right)$ vertices. The Crossing Lemma implies that the intersection graph of the edges of a dense topological graph is dense, so we have the following corollary.

Corollary 1. For every $x$-monotone drawing of a graph $G=(V, E)$ with $n$ vertices and $m=\Omega\left(n^{2}\right)$ edges, there are disjoint subsets $E_{1}, E_{2} \subset E$, each of size at least $\Omega\left(n^{2} / \log n\right)$, such that every edge in $E_{1}$ crosses all edges in $E_{2}$.

Corollary 1 is tight up to a constant factor by Theorem 2 Similar to Theorem 4 . Kolman and Matoušek KM04 proved an upper bound on the bisection width in terms of the pairwise crossing number and the $L_{2}$ norm of the degree sequence $d_{1}, d_{2}, \ldots, d_{n}$ :

$$
b(G)=O\left(\left(\sqrt{\text { pair-cr }(G)}+\sqrt{\sum_{i=1}^{n} d_{i}^{2}(G)}\right) \log n\right) .
$$

Using the same strategy as in the proof of Theorem 1, with the above mentioned tools instead of Theorems 3 and 4 , it is straightforward to establish the following.

Theorem 5. For every $x$-monotone drawing of a graph $G=(V, E)$ with $n$ vertices and $m \geq 3 n$ edges, there are disjoint subsets $E_{1}, E_{2} \subset E$, each of cardinality at least $m^{2} /\left(n^{2} \log ^{5+o(1)} n\right)$, such that every edge in $E_{1}$ crosses every edge in $E_{2}$.

In a special case, we can prove the same bound as in Theorem 1

Proposition 1. Given a bipartite graph $G$ with $n$ vertices and $m \geq 3 n$ edges, and an $x$-monotone drawing where the vertices of the two vertex classes lie on the lines $x=0$ and $x=1$, respectively, then the intersection graph of the edges contains a bi-clique of size $\Omega\left(m^{2} / n^{2}\right)$.

Proof. Consider the two dimensional partial order $\prec$ on the edges of $G$, where an edge $e_{1}$ is greater than another edge $e_{2}$ if and only if, for $j=0,1$ the endpoint of $e_{1}$ on the line $x=j$ lies above that of $e_{2}$. Two edges of $G$ must cross if they are incomparable by the partial order $\prec$. Also notice that there is an $x$-monotone drawing of $G$ with the vertices in the same position where two edges of $G$ cross if and only if they are incomparable under $\prec$. Indeed, this is done by drawing the edges as straight line segments.

By the Crossing Lemma, there are at least $\Omega\left(\mathrm{m}^{3} / \mathrm{n}^{2}\right)$ pairs of crossing edges in this straight-line drawing of $G$. Hence, there are at least $\Omega\left(m^{3} / n^{2}\right)$ pairs of incomparable elements under the partial order $\prec$. In [FPT07b] (Theorem 3), we prove that any incomparability graph with $m$ vertices and at least $d m$ edges 
contains a bi-clique of size at least $d$, so the intersection graph of the edges of $G$ must contain a bi-clique of size $\Omega\left(\mathrm{m}^{2} / \mathrm{n}^{2}\right)$.

Proposition 1 implies that Theorem 1 holds for $x$-monotone drawings if the vertex set lies in a bounded number of vertical lines. Indeed, an $x$-monotone drawing of a graph with all vertices contained in the union of $d$ vertical lines can be partitioned into $\left(\begin{array}{l}d \\ 2\end{array}\right) x$-monotone drawings of bipartite graphs with each vertex class lying on a vertical line.

Monotone properties. If a graph is drawn with at most $k$ crossings between any two edges and the graph has some additional property, then one may improve on the bound of Theorem 1

A graph property $\mathcal{P}$ is monotone if whenever a graph $G$ satisfies $\mathcal{P}$, every subgraph of $G$ also satisfies $\mathcal{P}$, and whenever graphs $G_{1}$ and $G_{2}$ satisfy $\mathcal{P}$, then their disjoint union also satisfies $\mathcal{P}$. The extremal number $\operatorname{ex}(n, \mathcal{P})$ denotes the maximum number of edges that a graph with property $\mathcal{P}$ on $n$ vertices can have. For graphs satisfying a monotone graph property, the bound (1) of the Crossing Lemma can be improved PST00. In particular, if $\mathcal{P}$ is a monotone graph property and $\operatorname{ex}(n, \mathcal{P})=O\left(n^{1+\alpha}\right)$ for some $\alpha>0$, then there exist constants $c, c^{\prime}>0$ such that for every graph $G$ with $n$ vertices, $m \geq c n \log ^{2} n$ edges, and property $\mathcal{P}$, the crossing number is at least $\operatorname{cr}(G) \geq c^{\prime} m^{2+1 / \alpha} / n^{1+1 / \alpha}$. Furthermore, if $\operatorname{ex}(n, \mathcal{P})=\Theta\left(n^{1+\alpha}\right)$, then this bound is tight up to a constant factor. A straightforward calculation shows, using the same strategy as in the previous section, the following strengthening of Theorem 1 .

Theorem 6. Let $\mathcal{P}$ be a monotone graph property such that ex $(n, \mathcal{P})=O\left(n^{1+\alpha}\right)$ for some $\alpha>0$. For every $k \in \mathbb{N}$, there exist positive constants $c$ and $c_{k}$ such that for any drawing of a graph $G=(V, E)$ satisfying property $\mathcal{P}$, having $n$ vertices and $m \geq c n \log ^{2} n$ edges, no two of which intersecting in more than $k$ points, there are disjoint subsets $E_{1}, E_{2} \subset E$, each of cardinality at least $c_{k}(m / n)^{1+1 / \alpha}$, such that every edge in $E_{1}$ crosses all edges in $E_{2}$.

Acknowledgment. We thank the anonymous referee for bringing a paper by Golumbic, Rotem, and Urrutia GRU83 to our attention.

\section{References}

[ACNS82] Ajtai, M., Chvátal, V., Newborn, M., Szemerédi, E.: Crossing-free subgraphs. In: Theory and Practice of Combinatorics. Mathematical Studies, vol. 60, pp. 9-12. North-Holland, Amsterdam (1982)

[D98] Dey, T.K.: Improved bounds for planar $k$-sets and related problems. Discrete Comput. Geom. 19, 373-382 (1998)

[ENR00] Elekes, G., Nathanson, M.B., Ruzsa, I.Z.: Convexity and sumsets. J. Number Theory 83, 194-201 (2000)

[E46] Erdős, P.: On sets of distances of $n$ points, Amer. Math. Monthly 53, 248250 (1946)

[F06] Fox, J.: A bipartite analogue of Dilworth's theorem, Order 23, 197-209 (2006) 
[FPT07a] Fox, J., Pach, J., Tóth, C.D.: Intersection patterns of curves, manuscript, Cf (2007), http://math.nyu.edu/ pach/publications/justcurves050907.pdf

[FPT07b] Fox, J., Pach, J., Tóth, C.D.: Turán-type results for partial orders and intersection graphs of convex sets, Israel J. Math. (to appear, 2007)

[GRU83] Golumbic, M.C., Rotem, D., Urrutia, J.: Comparability graphs and intersection graphs. Discrete Math. 43, 37-46 (1983)

[GM90] Gazit, H., Miller, G.L.: Planar separators and the Euclidean norm. In: Asano, T., Imai, H., Ibaraki, T., Nishizeki, T. (eds.) SIGAL 1990. LNCS, vol. 450, pp. 338-347. Springer, Heidelberg (1990)

[KT04] Katz, N.H., Tardos, G.: A new entropy inequality for the Erdős distance problem. In: Towards a theory of geometric graphs. Contemp. Math. AMS, vol. 342, pp. 119-126 (2004)

[KM04] Kolman, P., Matoušek, J.: Crossing number, pair-crossing number, and expansion. J. Combin. Theory Ser. B 92, 99-113 (2004)

[L84] Leighton, T.: New lower bound techniques for VLSI. Math. Systems Theory $17,47-70$ (1984)

[LT79] Lipton, R.J., Tarjan, R.E.: A separator theorem for planar graphs. SIAM J. Appl. Math. 36, 177-189 (1979)

[LPS88] Lubotzky, A., Phillips, R., Sarnak, P.: Ramanujan graphs. Combinatorica 8, 261-277 (1988)

[PRTT06] Pach, J., Radoičić, R., Tardos, G., Tóth, G.: Improving the Crossing Lemma by finding more crossings in sparse graphs. Discrete Comput. Geom. 36, 527-552 (2006)

[PSS96] Pach, J., Shahrokhi, F., Szegedy, M.: Applications of the crossing number. Algorithmica 16, 111-117 (1996)

[PS98] Pach, J., Sharir, M.: On the number of incidences between points and curves. Combin. Probab. Comput. 7(1), 121-127 (1998)

[PS01] Pach, J., Solymosi, J.: Crossing patterns of segments. J. Combin. Theory Ser. A 96, 316-325 (2001)

[PST00] Pach, J., Spencer, J., Tóth, G.: New bounds on crossing numbers. Discrete Comput. Geom. 24(4), 623-644 (2000)

[PT00] Pach, J., Tóth, G.: Which crossing number is it anyway? J. Combin. Theory Ser. B 80, 225-246 (2000)

[PT02] Pach, J., Tardos, G.: Isosceles triangles determined by a planar point set. Graphs Combin. 18, 769-779 (2002)

[PT06] Pach, J., Tóth, G.: Comment on Fox News. Geombinatorics 15, 150-154 (2006)

[ST01] Solymosi, J., Tóth, C.D.: Distinct distances in the plane. Discrete Comput. Geom. 25, 629-634 (2001)

[STT02] Solymosi, J., Tardos, G., Tóth, C.D.: The $k$ most frequent distances in the plane. Discrete Comput. Geom. 28, 639-648 (2002)

[S97] Székely, L.A.: Crossing numbers and hard Erdős problems in discrete geometry. Combin. Probab. Comput. 6, 353-358 (1997)

[ST83] Szemerédi, E., Trotter, W.T.: Extremal problems in discrete geometry. Combinatorica 3, 381-392 (1983)

[TV06] Tao, T., Vu, V.: Additive combinatorics. Cambridge Studies in Advanced Mathematics, 105. Cambridge University Press, Cambridge (2006)

[V05] Valtr, P.: On the pair-crossing number. In: Combinatorial and computational geometry, vol. 52, pp. 569-575. MSRI Publications, Cambridge Univ. Press (2005) 